\title{
New spectroscopic binaries among nearby stars ${ }^{\star}$
}

\author{
R. Wichmann ${ }^{1, \star \star}$, J. H. M. M. Schmitt ${ }^{1}$, and S. Hubrig ${ }^{2}$ \\ 1 Hamburger Sternwarte, Gojenbergsweg 112, 21029 Hamburg, Germany \\ ${ }^{2}$ European Southern Observatory, Karl-Schwarzschild-Str. 2, 85748 Garching bei München, Germany
}

Received 17 October 2002 / Accepted 20 December 2002

\begin{abstract}
In the course of surveying a large number of nearby ( $\approx 50 \mathrm{pc}$ or less) stars for indicators of youth, we have discovered a number of hitherto unknown spectroscopic binaries. Here we present a list of these new binaries with pertinent data. Two of these (HD 143705 and HD 89959) have been observed six times each, and their respective radial velocity curves are discussed.
\end{abstract}

Key words. Stars: binaries: general

\section{Introduction}

We have recently performed an all-sky survey for nearby young stars, the results of which were published in Wichmann et al. (2003). The candidate sample for this survey was obtained by cross-correlating the ROSAT All-Sky Survey (RASS) with the TYCHO catalogue (ESA 1997) and selecting stars with $B-V>0.54$ (i.e. F8 and later) with (TYCHO) parallaxes larger than their respective $3.5 \sigma$ errors. In addition, stars very far above the main sequence (giants) and very far below (erroneous parallaxes) were discarded, resulting in a total sample size of 754 stars. The region around the main sequence from which candidate stars were drawn is depicted in Fig. 1 in Wichmann et al. (2003). For internal consistency, in the original selection procedure only TYCHO parallaxes were used to determine the location of the stars in the HR diagram. However, in the later analysis HIPPARCOS (ESA 1997) parallaxes were used whenever available. In particular, all stars presented here except HD 89959 have HIPPARCOS parallaxes.

The spectroscopic follow-up investigations of 748 stars out of this sample were geared towards the detection of signs of youth such as lithium $\lambda 6708$ absorption and/or rapid rotation. However, they also led to the serendipitous discovery of a sizeable number of hitherto unknown spectroscopic binaries presented here. All of them are double-lined (SB2) binaries, which are interesting for orbit and mass determinations. In general each star in our survey was observed only once, so that we are

Send offprint requests to: $\mathrm{R}$. Wichmann,

e-mail: rwichmann@hs. uni-hamburg.de

* Based on observations collected at the European Southern Observatory, Chile (ESO No. 62.I-0650, 66.D-0159(A), 67.D-0236(A)).

$\star \star$ Visiting Astronomer, Kitt Peak National Observatory, National Optical Astronomy Observatory, which is operated by the Association of Universities for Research in Astronomy, Inc. (AURA) under cooperative agreement with the National Science Foundation. not able to detect SB1 binaries. As the detection of these SB2 binaries was not the main goal of our survey, no follow-up observations have been carried out or are currently planned by us.

\section{Observations}

Observations were carried out at three different observatories: ESO (La Silla, Chile), KPNO (Kitt Peak, USA), and DSAZ (Calar Alto, Spain). At ESO, the $1.52 \mathrm{~m}$ telescope with the FEROS echelle spectrograph $(R=48000)$ was used. Observations at KPNO were performed using the 0.9 m Coude Feed telescope with the Coudé spectrograph (camera 5, grating A, Ford $3 \mathrm{~K} \times 1 \mathrm{~K} \mathrm{CCD}, R=25000)$. At the DSAZ, we used the FOCES echelle spectrograph $(R=40000)$ on the $2.2 \mathrm{~m}$ telescope. The observing date and instrument for each star presented here is listed in Table 1.

In addition to our target stars, usually two radial velocity standard stars were observed each night. Data reduction of the KPNO and DSAZ observations was performed using standard IRAF tasks. The ESO data were reduced online during the observations using the data reduction pipeline provided by the FEROS team.

\section{Results}

Radial velocities for all stars were measured by crosscorrelating them against radial velocity standard stars observed during the same run using the IRAF "xcsao" task. The identification of binary stars was based on both the cross-correlation function and the visual appearance of the spectrum. In almost all of our new SB2 the spectral lines are clearly doublelined (triple-lined in the case of HD 10800), with the exception of HD 40705 where the lines show a marked asymmetry in the profiles (see Fig. 1).

In Table 1, for each new spectroscopic binary, we list the velocities of both components at the time of observation, and 


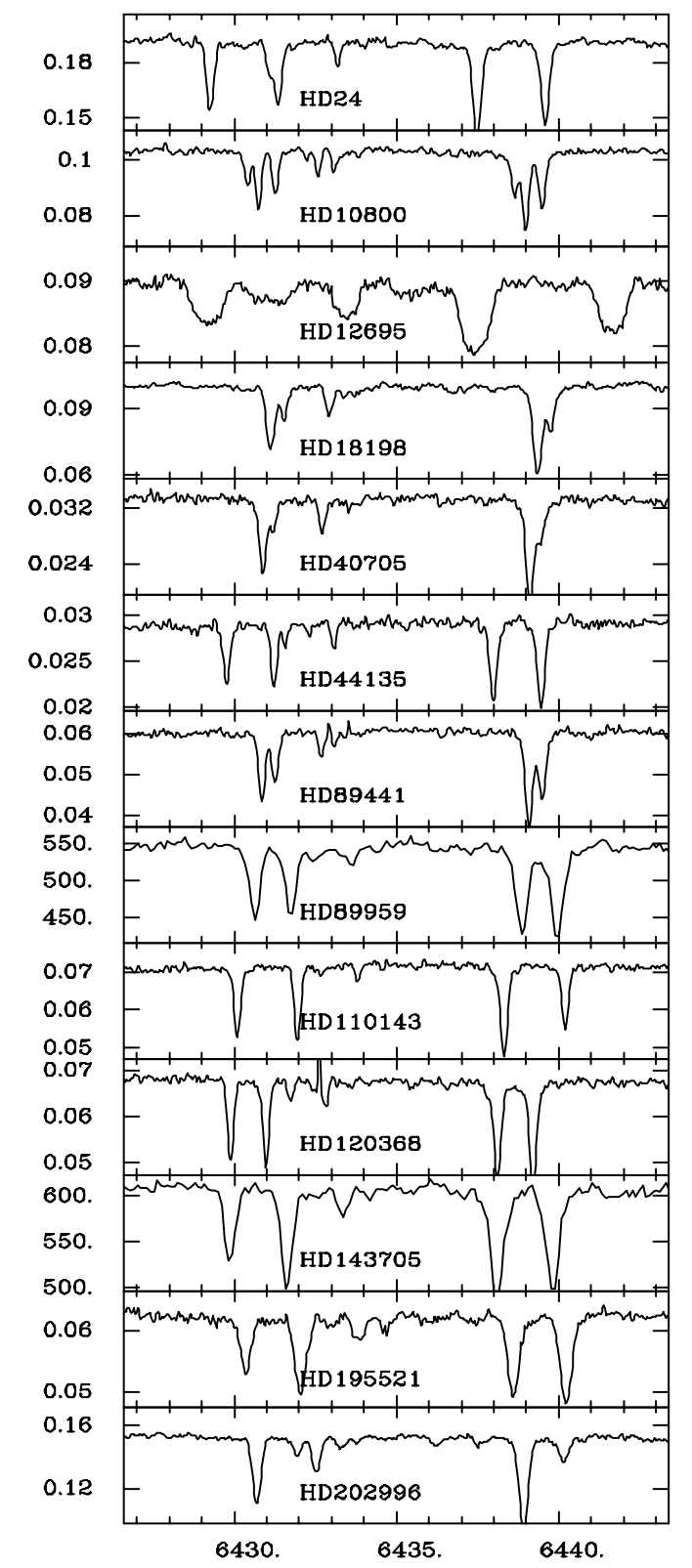

Fig. 1. Spectra of new SB2 stars. Shown is the region from $6427 \AA$ to $6443 \AA$, comprising the two strong lines Fe I $\lambda 6430.824 \AA$, Ca I $\lambda 6439.075 \AA$, as well as a weak Fe II line $(\lambda 6432.680 \AA)$.

indicate which of them is the brighter one (only if there is a marked difference).

\subsection{Remarks on individual stars}

Most stars have hitherto not been recognized as binaries except for HD 10800, HD 12695 and HD 18198 which we discuss below.

\subsubsection{HD 10800 (HR 512)}

This is a G2V star with a $V$ magnitude of $5.89 \mathrm{mag}$ and a HIPPARCOS parallax of $36.86 \pm 0.52$ mas. The star is noted as having variable radial velocity in Buscombe \& Williams (1958). However, its seems to have never been confirmed as an SB2. Our data indicate that HD 10800 is a spectroscopic triple system.

\subsubsection{HD 12695}

This F8 star with $V=8.07$ mag and a HIPPARCOS parallax of $15.49 \pm 0.69$ mas shows a rather high X-ray activity, comparable to the 10 bona-fide zero-age main sequence stars within our complete sample. It is also a rapid rotator $\left(\approx 26 \mathrm{~km} \mathrm{~s}^{-1}\right)$. We did not detect the lithium line. However, unless the orbit is highly eccentric, this is probably a close binary with a separation of a few solar radii only, where activity might be driven by the rapid rotation caused by synchronization due to tidal forces.

Cutispoto et al. (2002) measure radial velocity variations, but note that "it (the star) is reported as SB2 by CORAVEL; however ... there is no evidence for the secondary component in our spectra". In our spectrum the SB2 nature is clearly visible, and the line equivalent widths of both components only differ by some 15 per cent.

\subsubsection{HD 18198}

This star is a visual binary with the primary classified as K0III. Fabricio \& Makarov (2000) have determined $B_{\mathrm{T}}=8.70 \mathrm{mag}$, $V_{\mathrm{T}}=8.03 \mathrm{mag}$ for the primary, and $B_{\mathrm{T}}=10.40 \mathrm{mag}, V_{\mathrm{T}}=$ $9.61 \mathrm{mag}$ for the secondary, where the subscript "T" refers to the TYCHO photometric system. These measurements correspond to $V=7.97 \mathrm{mag}, B-V=0.67 \mathrm{mag}$ for the primary and $V=9.54 \mathrm{mag}, B-V=0.79 \mathrm{mag}$ for the secondary (ESA 1997, Eq. (1.3.20)). The HIPPARCOS parallax is $14.84 \pm 1.13$ mas. Speckle observations by Hartkopf et al. (1996) show a separation of $\rho=0.43^{\prime \prime}$. At the Hipparcos distance of $67 \mathrm{pc}$, this corresponds to $29.5 \mathrm{AU}$. If the two SB2 components in our spectrum correspond to the two visual components, a total mass of some $17 M_{\odot}$ would be required e.g. for a circular orbit with inclination $i=90^{\circ}$ to explain the observed $\Delta V_{\text {rad }}$ of the two SB2 components.

However, the $V$ magnitude of the HD 18198 primary corresponds to an absolute $V$ magnitude of about $M_{V} \simeq 3.83$, This (and also the $B-V$ value of $0.67 \mathrm{mag}$ ) is inconsistent with a KOIII spectral classification. It would be consistent with a young $\approx 1.2-1.4 M_{\odot}$ star shortly before reaching the ZAMS (Siess et al. 2000), or a slightly evolved star of about 1.0 $1.2 M_{\odot}$ (Vandenberg 1985). We therefore conclude that the two SB2 components in our spectrum do not correspond to the visual components, but rather that HD 18198 is a triple system.

In addition, the star shows an appreciable $\mathrm{Li} \lambda 6708$ equivalent width (110 m $\AA$ for both SB2 components together). This is less than the upper limit of the Pleiades for the same $B-V$, but may indicate that the star could be quite young. 
Table 1. New spectroscopic binaries. This table contains those new binaries which have been observed once only. Listed are the HD number of each star (an asterix indicates additional remarks in the text), the HIP number, the (MJD) date of observation the heliocentric radial velocities $\left[\mathrm{km} \mathrm{s}^{-1}\right]$ measured, a flag telling which component is the primary, $\log \left(L_{x}\right)\left[\mathrm{erg} \mathrm{s}^{-1}\right], \log \left(L_{x} / L_{\mathrm{bol}}\right)$, and $v \sin i\left[\mathrm{~km} \mathrm{~s}^{-1}\right]$. Errors for $V_{\text {rad }}$ are typically $1 \mathrm{~km} \mathrm{~s}^{-1}$ for most stars, but larger (3-5 km s-1) for HD 12695 (broad lines), and for HD 40705 (lines blended). The error for $v \sin i$ is about $10 \%$ for HD 12695, but larger (about 3-4 $\mathrm{km} \mathrm{s}^{-1}$ ) for the other stars, which are at the measurement limit. All stars in this list were observed with FEROS.

\begin{tabular}{|c|c|c|c|c|c|c|c|c|c|}
\hline HD number & HIP number & Date & $V_{\text {rad,1 }}$ & $V_{\mathrm{rad}, 2}$ & $V_{\mathrm{rad}, 3}$ & Primary & $\log \left(L_{x}\right)$ & $\log \left(L_{x} / L_{\mathrm{bol}}\right)$ & $v \sin i$ \\
\hline 24 & 425 & 51847.106 & -74.4 & 23.0 & & 1 & 28.14 & -4.43 & $<6.0$ \\
\hline $10800^{*}$ & 7601 & 51845.247 & -20.6 & -5.4 & 18.4 & 2 & 29.58 & -4.72 & $<6.0$ \\
\hline $12695^{*}$ & 9499 & 51846.222 & -78.2 & 121.6 & & 1 & 29.16 & -3.51 & 25.8 \\
\hline $18198^{*}$ & 13498 & 51847.307 & 12.9 & 31.2 & & 1 & 28.01 & -4.76 & 7.5 \\
\hline 40705 & 28311 & 52021.015 & 2.0 & 16.0 & & 1 & 28.03 & -4.66 & $<6.0$ \\
\hline 44135 & 30001 & 52020.010 & -51.6 & 16.7 & & 2 & 28.05 & -4.63 & $<6.0$ \\
\hline 89441 & 50489 & 52020.134 & -0.4 & 17.8 & & 1 & 28.61 & -4.51 & $<6.0$ \\
\hline 110143 & 61840 & 52020.168 & -36.2 & 51.4 & & $?$ & 28.40 & -4.85 & $<6.0$ \\
\hline 120368 & 67458 & 51265.352 & -46.0 & 5.4 & & 2 & 28.42 & -4.65 & $<6.0$ \\
\hline 195521 & 101432 & 52021.342 & -23.4 & 52.4 & & 2 & 29.35 & -4.26 & 9.7 \\
\hline 202996 & 105391 & 51847.006 & -7.6 & 50.3 & & 1 & 28.18 & -4.90 & 6.2 \\
\hline
\end{tabular}

Table 2. Radial velocities for HD 89959 (BD+41 2078), observed with the Coudé spectrograph on KPNO. Listed are the (MJD) date of observation, and the heliocentric radial velocities (in $\mathrm{km} \mathrm{s}^{-1}$ ) measured. Errors are typically $1 \mathrm{~km} \mathrm{~s}^{-1}$.

\begin{tabular}{rrr}
\hline \hline Date & $V_{\text {rad,1 }}$ & $V_{\text {rad,2 }}$ \\
\hline 51264.249 & -51.8 & 42.3 \\
51265.231 & -53.6 & 44.1 \\
51266.294 & -26.4 & 18.2 \\
51267.254 & -4.8 & -4.8 \\
51268.162 & -3.8 & -3.8 \\
51269.345 & 20.5 & -29.1 \\
\hline
\end{tabular}

\subsection{Radial velocity curves of HD 89959 and HD 143705}

The SB2 nature of HD 89959 and HD 143705 was noted during the preliminary data reduction at the telescope. As this was early during a week-long run, we decided to follow their radial velocity curves. The resulting data are presented in Tables 2 and 3 .

We attempted to fit orbital solutions to the radial velocity curve of HD 143705 (see Tables 4, 5 and Fig. 2). When we include all our data, the best fit is still not acceptable, because the rms error of $3.6 \mathrm{~km} \mathrm{~s}^{-1}$ is significantly larger than the typical observational error of about $1 \mathrm{~km} \mathrm{~s}^{-1}$. However, the most outlying point is the one closest to conjunction, i.e. the least precisely measured (because of line blending). Removing this data point (Table 5) improves the fit notably, and brings the rms error in line with the observational error.

As only few data points are available, and as the data do not cover a full orbital period, the errors in the derived parameters are relatively large, and the solution should be regarded as tentative only. We do not present an orbital solution for HD 89959,
Table 3. Radial velocities for HD 143705 (HIP 78450), observed with the Coude spectrograph on KPNO. Listed are the (MJD) date of observation, and the heliocentric radial velocities (in $\mathrm{km} \mathrm{s}^{-1}$ ) measured. Errors are typically $1 \mathrm{~km} \mathrm{~s}^{-1}$.

\begin{tabular}{rrr}
\hline \hline Date & $V_{\text {rad, } 1}$ & $V_{\text {rad,2 }}$ \\
\hline 51264.47329861 & -39.4 & 50.6 \\
51265.39203704 & -15.0 & 27.5 \\
51266.48636574 & 5.8 & 5.8 \\
51267.45549769 & 42.8 & -30.5 \\
51268.39733796 & 56.5 & -43.2 \\
51269.39416667 & 47.3 & -33.9 \\
\hline
\end{tabular}

because due to an unfavourable phase coverage no unique fit could be achieved ${ }^{1}$.

HD 143705 is classified as a G0V star $(V=7.96 \mathrm{mag})$ with a HIPPARCOS parallax of $16.16 \pm 0.98$ mas. Our orbital solution yields a mass ratio of $\approx 1$, i.e. both components have equal masses (within the errors). The bolometric luminosity of the system is $M_{\text {bol }}=3.82$, i.e. with identical components each would have $M_{\text {bol }} \approx 4.57$, which is more in line with a G2V spectral type.

With our orbit solution (assuming an eccentricity of $e=$ 0.13 ), the major axes would be $a_{1,2} \sin i \approx 0.04 \mathrm{AU}$, and the mass functions are $M_{1,2} \sin ^{3} i \approx 0.47$. Assuming that the system comprises two almost identical G2V stars, $\sin i$ would be approximately 0.78 , and the separation about $0.1 \mathrm{AU}$. However, $a \sin i$ depends on $\sqrt{1-e^{2}}$, and $M \sin ^{3} i$ on $\left(1-e^{2}\right)^{3 / 2}$. Because $e$ is not well constrained in our fit (due to the sampling, see Fig. 2), both values are rather uncertain at the moment.

\footnotetext{
1 The program velocity written to fit the radial velocity curve is available via anonymous ftp from

ibiblio.org/pub/Linux/science/astronomy/
} 


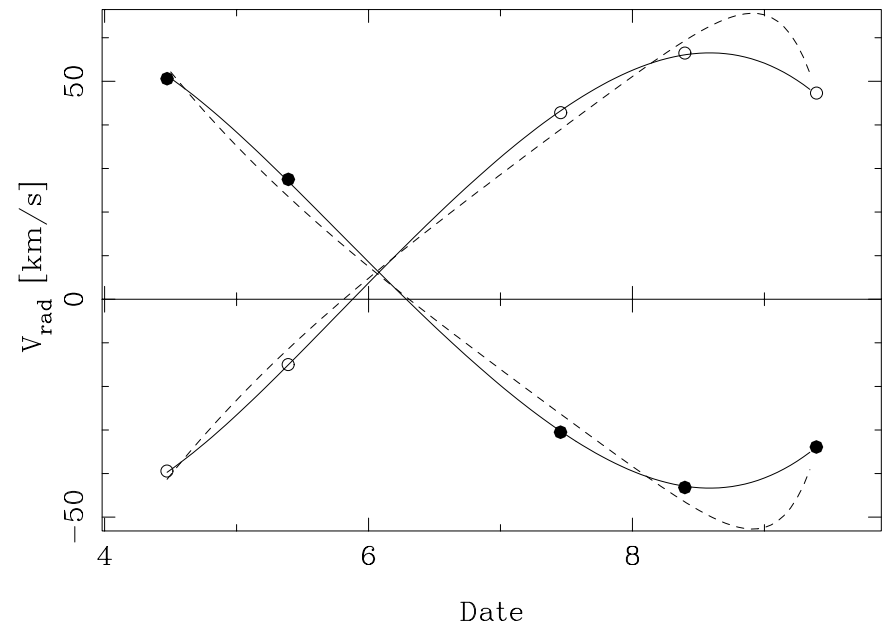

Fig. 2. Radial velocity curve of HD 143705. The solid lines show our best-fit solution (Table 5). We also plot a best-fit solution for $e$ fixed at 0.5 (dashed line) to show that due to our limited sampling, $e$ is not well constrained.

Table 4. Orbital solution for HD 143705 (all data). This fit results in an rms error of $3.6 \mathrm{~km} \mathrm{~s}^{-1}$, in excess of the observational error for most of the data points. Errors are determined from the covariance matrix.

\begin{tabular}{crlll}
\hline \hline Parameter & Result & & & \\
\hline$\gamma$ & 6.1 & \pm & $0.3 \mathrm{~km} \mathrm{~s}^{-1}$ & \\
$K_{1}$ & 51.1 & \pm & $2.6 \mathrm{~km} \mathrm{~s}^{-1}$ & \\
$K_{2}$ & 50.2 & \pm & $2.7 \mathrm{~km} \mathrm{~s}^{-1}$ & \\
$e$ & 0.17 & - & $0.17 \quad+$ & 0.42 \\
$\omega$ & 1.36 & \pm & $0.25 \mathrm{rad}$ & \\
$P$ & 8.01 & \pm & $0.73 \mathrm{~d}$ & \\
$T$ & 51261.99 & \pm & $0.18 \mathrm{MJD}$ & \\
\hline
\end{tabular}

\section{Discussion}

This was an all-sky survey without any preference for any of the hemispheres. We regard the preferential detection of new SB2s on the southern hemisphere (11 vs. 2) as an indication that this hemisphere is not as well studied as the northern one (the two northern SB2 are HD 89959 and HD 143705). There is no significant bias towards the southern hemisphere in the original sample of selected candidates, therefore the larger number of new detection on this hemisphere is not an artefact of the selection procedure.
Table 5. Orbital solution for HD 143705 (excluding the data point at conjugation). This fit results in an rms error of $0.4 \mathrm{~km} \mathrm{~s}^{-1}$, in line with the observational error. Errors are determined from the covariance matrix.

\begin{tabular}{crlll}
\hline \hline Parameter & Result & & & \\
\hline$\gamma$ & 6.2 & \pm & $0.2 \mathrm{~km} \mathrm{~s}^{-1}$ & \\
$K_{1}$ & 52.7 & \pm & $5.2 \mathrm{~km} \mathrm{~s}^{-1}$ & \\
$K_{2}$ & 51.8 & \pm & $5.2 \mathrm{~km} \mathrm{~s}^{-1}$ & \\
$e$ & 0.13 & - & $0.13 \quad+$ & 0.42 \\
$\omega$ & 1.91 & \pm & $0.44 \mathrm{rad}$ & \\
$P$ & 8.43 & \pm & $0.87 \mathrm{~d}$ & \\
$T$ & 51262.37 & \pm & $1.24 \mathrm{MJD}$ & \\
\hline
\end{tabular}

We further note that most stars studied in our survey are relatively nearby $(d \leq 100 \mathrm{pc})$. Taking our numbers for HD 143705 as an example, the separation of the components is a little under 1 mas, so that directly resolving HD 143705 will provide a challenge even for the ESO VLTI. Nevertheless, in view of the forthcoming availability of interferometric telescopes, future study of these new SB2s is worthwhile, because these systems will eventually provide fundamental astronomical data such as stellar masses and radii.

Acknowledgements. This project has been supported by grants from the Deutsche Forschungsgemeinschaft (DFG Schwerpunktprogramm "Physics of star formation").

This research has made use of the SIMBAD database, operated at CDS, Strasbourg, France.

\section{References}

Buscombe, W., \& Morris, P. M. 1958, MNRAS, 118, 609

Cutispoto, G., Pastori, L., Pasquini, L., et al. 2002, A\&A, 384, 491

ESA 1997, The Hipparcos and Tycho Catalogues, ESA SP-1200

Fabricio, C., \& Makarov, V. V. 2000, A\&A, 356, 141

Hartkopf, W. I., Mason, B. D., McAlister, H. A., et al. 1996, AJ, 111, 936

Siess, L., Dufour, E., \& Forestini, M. 2000, A\&A, 358, 593

Vandenberg, D. A. 1985, ApJS, 58, 711

Wichmann, R., Schmitt, J. H. M. M., \& Hubrig, S. 2003, A\&A, 399, 983 